\title{
Effects of Mode Dispersion on Cross-phase modulation on Mode Division Multiplexed Optical Fibers
}

\author{
Reinhardt Rading ${ }^{(1,2)}$ \\ ${ }^{(1)}$ Institut Polytechnique de Paris \\ ${ }^{(2)}$ University of Parma
}

\begin{abstract}
The concept of mode division multiplexing also known as space division multiplexing was introduced as an alternative to combat the approaching capacity crunch in single mode fibers. Just like single mode fibers, space division multiplexed fibers will experience non-linearities at a different level and studies have shown that some linear effects can be beneficial in combating the nonlinear interferences. This study aims to identify the benefits accrued when these linear effects are implemented by exploring the already existing models defined in the literature.
\end{abstract}

Index Terms-Cross-phase modulation, Mode division multiplexing, Nonlinear interference, Optical fibers.

\section{INTRODUCTION}

Network traffic continues to grow at more than $30 \%$ per year and is even expected to grow further with more people and devices being connected to the internet. During this COVID19 period, we just witnessed unpredictable [1] increase in data demands that are stressing the existing optical fiber networks. Previously thought as having a limitless capacity, single mode fibers (SMF) currently provides an infrastructure for transmitting hundreds of gigabytes and studies have shown that there is a capacity limit [2] resulting from the non-linear response of fiber optic systems and that we are approaching a SMF capacity crunch [3] hence the need for new technologies to enhance the capacity of the existing optical fiber systems.

Space division multiplexing (SDM) has received a lot of attention from researchers as an alternative solution and just like in SMF fibers, its throughput are influenced by nonlinearities. Studies has shown that strong mode coupling and mode dispersion are beneficial in combating these interferences. For example, strong mode coupling is seen as beneficial in reducing the delay delay spread factor between the modes and thus a reduced complexity at the receiver and a reduced mode dependent loss during propagation [4]. Studies has shown further that a huge mode dispersion tends to reduce the non-linear Kerr effects and hence an improved optical system performance.

In this study, $\mathrm{i}$ have shown the interplay between mode dispersion and non-linear interference in SDM fiber optic systems. This study aims to show that there is a better system performance especially in combating cross-phase modulation when strong mode dispersion is allowed on the optical link. Furthermore, this study will show that by varying other optical fiber system parameters with strong mode dispersion, the quality of transmission in fiber optic systems is improved.

\section{THEORY}

Analysing non-linear effects in SDM systems has become challenging since the introduction of the SDM concept. This is because of the several non-linear coefficients involved in the coupled non-linear Schroedinger equation. Several models have been developed to navigate around this predicament and predict the performance of a such system; one of such model is the Gaussian noise model [5] initially used for SMF and extended to accommodate SDM in [6].

While mode dispersion in single mode fibers exists in form of polarization mode dispersion and can be neglected, mode dispersion in multimode or multicore fibers cannot be neglected because it might be beneficial in higher values. The omission of mode dispersion in modelling the SDM Gaussian model in [6] galvanized Serena in [7] to improve the model accommodate it.

Consider the propagation of two quasi degenerate mode groups in an SDM fiber, denoting one group as group a and the other group as group b. Modes in each group are categorized as having nearly equal propagation constant and sums to $2 N_{a}$ and $2 N_{b}$, a factor that includes the two polarization of each mode.The propagation of each mode group is described by:

$$
\begin{aligned}
\frac{d \vec{E}_{a}}{d Z}=-\frac{\alpha}{2} \vec{E}_{a}+i \beta_{a} \vec{E}_{a}- & \beta_{a}^{\prime} \frac{d \vec{E}_{a}}{d t}-\mathbf{B}_{a} \frac{d \vec{E}_{a}}{d t}-i \frac{\beta_{a}^{\prime \prime}}{2} \frac{d^{2} \vec{E}_{a}}{d t^{2}} \\
& +i \gamma\left(K_{a a}\left|\vec{E}_{a}\right|^{2}+K_{a b}\left|\vec{E}_{b}\right|^{2}\right) \vec{E}_{a}
\end{aligned}
$$

$$
\begin{aligned}
\frac{d \vec{E}_{b}}{d Z}=-\frac{\alpha}{2} \vec{E}_{b}+i \beta_{b} \vec{E}_{b} & -\beta_{b}^{\prime} \frac{d \vec{E}_{b}}{d t}-\mathbf{B}_{b} \frac{d \overrightarrow{E_{b}}}{d t}-i \frac{\beta_{b}^{\prime \prime}}{2} \frac{d^{2} \vec{E}_{b}}{d t^{2}} \\
& +i \gamma\left(K_{b a}\left|\vec{E}_{a}\right|^{2}+K_{b b}\left|\vec{E}_{b}\right|^{2}\right) \vec{E}_{b}
\end{aligned}
$$

where $\mathbf{B}_{a}$ and $\mathbf{B}_{b}$ are $2 \mathrm{X} 2$ matrix that describes the birefringence experienced within the fiber. $\beta_{a}^{\prime \prime}$ and $\beta_{b}^{\prime \prime}$ are the chromatic 


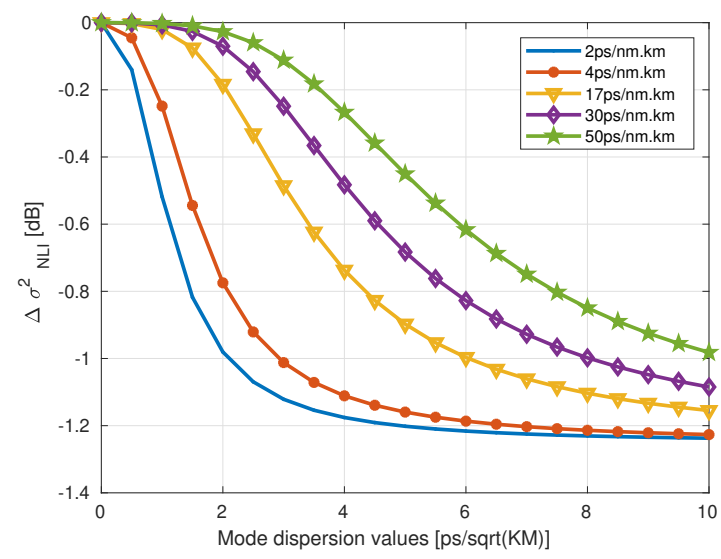

Fig. 1. Variance of XPM with dispersion using the Gaussian (GN) model.

dispersion coefficient, $\alpha$ is the attenuation constant and $\beta_{a}^{\prime}$ and $\beta_{b}^{\prime}$ are the inverse group velocity.

During the propagation if we consider a mode division multiplexed-wavelength division multiplexing(MDM-WDM) system as shown in equation 6 and 7 of [8], the two groups will experience intra-group and inter-group non-linear interference as shown in equation 3 and 4 respectively and proved in[8]. The non-linear interference experienced are self-phase modulation(SPM) and cross-phase modulation (XPM)

$$
\begin{gathered}
i \gamma K_{a a}\left({\overrightarrow{E^{\dagger}}}_{a, l}+\vec{E}_{a, l-m}+\eta_{l, m}{\overrightarrow{E^{\dagger}}}_{a, l} \vec{E}_{a, l-m} \vec{E}_{a, m}\right) \\
i \gamma K_{a b}{\overrightarrow{E^{\dagger}}}_{b, l} \vec{E}_{b, m} \vec{E}_{a, l-m}
\end{gathered}
$$

Considering the non-linear interference especially focusing on XPM in SDM systems as proved in equation 18 and 19 in [8] and respecting the conditions as set in [7], the variance of XPM in SDM case in the presence of mode dispersion becomes

$$
\begin{gathered}
\delta_{X P M}^{2}=\frac{2 N+1}{2 N}\left\{(2 N+1) \delta_{X P M 1}^{2}(\alpha)\right. \\
\left.+\frac{(2 N-1)\left(\alpha+\frac{\omega^{2} \mu^{2}}{N}\right)}{\alpha} \delta_{X P M 1}^{2}\left(\alpha+\frac{\omega^{2} \mu^{2}}{N}\right)\right\}
\end{gathered}
$$

where $\delta_{X P M 1}^{2}$ is the XPM variance experienced in the scalar case( single mode case), $\mu$ is a function of mode dispersion, $\mathrm{N}$ is the number of group modes and $\omega$ is the channel spacing.

\section{Simulation AND Results}

Having extended the GN model for SDM in [7] to include mode dispersion, $\mathrm{i}$ simulated the model to determine the system response in terms of performance when mode dispersion and other optical fiber system are varied. Initially, i considered a fiber system with a dispersion value of $17 \frac{\mathrm{ps}}{\mathrm{nm} \cdot \mathrm{km}}$, attenuation of $0.2 \mathrm{~dB} / \mathrm{km}$ and $\mathrm{N}$-a varied number of group of modes ( $2 \mathrm{~N}$ spatial modes), starting with $\mathrm{N}=2$. I considered two channels being transmitted in the system with the channel of interest having a power of $-30 \mathrm{~dB}$ and the interfering channel having OdB; this was to suppress the effect of SPM in the

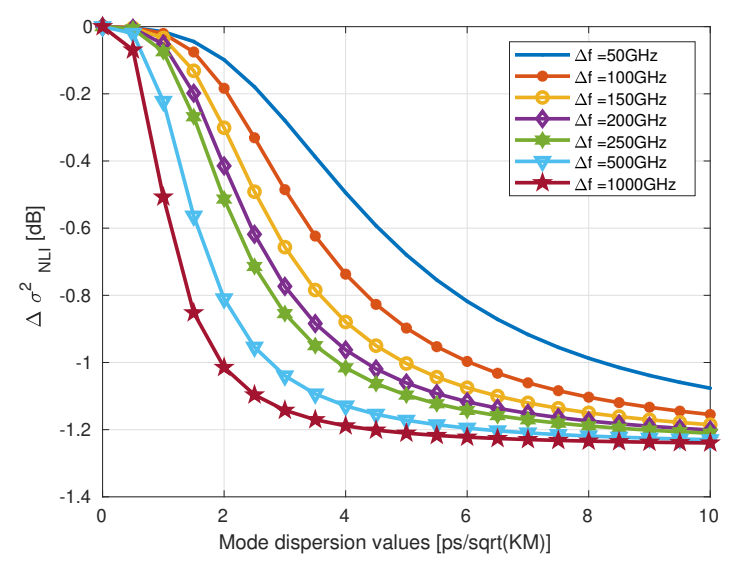

Fig. 2. Variance of XPM with channel spacing using the GN model..

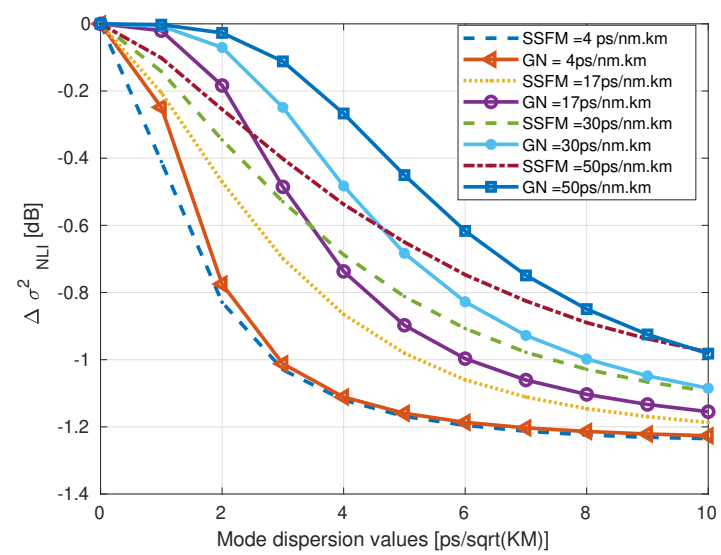

Fig. 3. Solid lines with symbols are the Gaussian Model while the dashed lines portrays the waveplate-SSFM model.

channel of interest and only analyse the effect of XPM from the interfering channel. I used Gaussian distributed symbols with 16384 symbols at a symbol rate of 49GB with an initial channel spacing of $100 \mathrm{GHz}$. The spacing was varied later to understand the system response. Amplified spontaneous emission was neglected in the simulation as the goal in this study was to identify the influence of mode dispersion on nonlinear interference.

From figure 1, we observed a decrease in XPM variance with an increase the mode dispersion value. An increase in XPM variance was experienced when the values of fiber dispersion was increased.

In figure 2, we observed that the XPM variance reduced with an increase in the channel spacing value. A further reduction was observed when the mode dispersion value was increased.

To confirm my results, i repeated the simulation using the wave-plate [9]-split step Fourier method (SSFM). The waveplate SSFM results converges with the result of this study as shown in figure 3 and figure 4. 


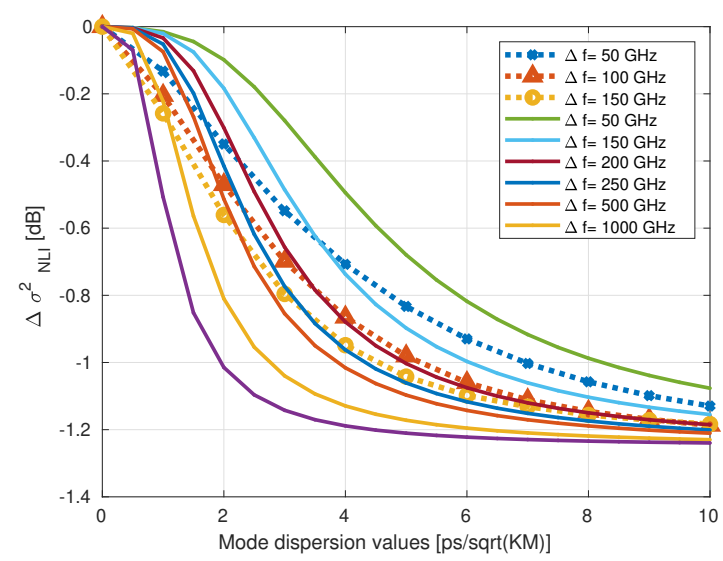

Fig. 4. Figure confirming the channel spacing results by showing both the Gaussian model and the waveplate-SSFM simulation.The lines with symbols are the wave-plate-SSFM model while the solid lines are the Gaussian models as previously shown in figure 2 .

\section{CONCLUSION}

Cross-phase modulation is one of the main interferences that reduces the throughput of space division multiplexed optical fibers. This study has shown that XPM variance can be reduced by increasing the mode dispersion in the optical system.

While this study made an assumption of no existence of intra-modal dispersion, $\mathrm{i}$ believe this study makes a foundation for future research where the existence of both intra and intermodal dispersion will be explored.

\section{REFERENCES}

[1] "Network traffic insights in the time of COVID-19: April 9 update"https://www.nokia.com/blog/network-traffic-insights-time-covid-19april-9-update.

[2] P. Mitra and J. Stark 'Nonlinear limits to the information capacity of optical fibre communications 'Letters to Nature, Vol 411, June 2001

[3] A. Chralyvy, "The Coming Capacity Crunch", Bell Labs, AlcatelLucent, USA-ECOC 2009, 20-24 September, 2009, Vienna, Austria.

[4] C. Antonelli, M. Shtaif, and A. Mecozzi, "Modeling of non-linear propagation in Space-Division Multiplexed fiber optic transmission," J. Lightw. Technol., vol. 34, no. 1, pp.36-54, 2016

[5] P. Poggiolini, G. Bosco, A. Carena, V. Curri, Y. Jiang, and F. Forghieri, "The GN-Model of Fiber Non-Linear Propagation and its Applications," J. Lightw. Technol., vol. 32, no. 4, pp. 694-721, 2014.

[6] G. Rademacher and K. Petermann, "Nonlinear Gaussian Noise Model for Multimode Fibers with Space-Division Multiplexing," J. Lightw. Technol, vol. 34, no. 9, pp. 2280-2287, 2016.

[7] P. Serena, C.Lasagni, A. Bononi , C. Antonelli, and A. Mecozzi, "A Model of the Nonlinear Interference in Space-Division Multiplexed Systems with Arbitrary Modal Dispersion"- ECOC 2021

[8] C. Antonelli, M. Shtaif, and A. Mecozzi, "Nonlinear interference noise in space-division multiplexed transmission through optical fibers" Optics Express, Vol. 25, No. 12 ,pp.13055-13075, 2017

[9] F. Curti,B.Diano, Q.Mao, F.Matera, and G. Someda "Concatenation of Polarisation Dispersion in Single Mode Fibers", Electron Lett., vol 25. pp 290-292. 1989 